\title{
Organochlorine Pesticide Residues in Caudal Scutes of Belize Morelet's Crocodiles (Crocodylus moreletii)
}

\author{
Brie D. Sherwin, ${ }^{1,2}$ Joseph F. Mudge,${ }^{1}$ Jaclyn E. Cañas-Carrell,${ }^{1}$ Heather A. Lanza, ${ }^{1}$ Thomas R. Rainwater, ${ }^{1}$ Steven \\ G. Platt, ${ }^{3}$ Scott T. McMurry, ${ }^{4}$ and Todd A. Anderson ${ }^{1}$ \\ ${ }^{1}$ Department of Environmental Toxicology, The Institute of Environmental and Human Health (TIEHH), Texas Tech University, Lubbock, Texas USA \\ ${ }^{3}$ Wildife Conservation Society, 2300 Southern Blvd., Bronx, New York USA \\ ${ }^{4}$ Department of Integrative Biology, Oklahoma State University, Stillwater, Oklahoma USA
}

\begin{abstract}
Historically, Belize has used large quantities of organochlorine (OC) pesticides for agriculture and disease-vector control, yet few tools exist for noninvasive assessment of OC contaminant loads in Belize wildlife. Crocodile caudal (tail) scutes are clipped as a marking technique in wildlife management programs and may also have utility as a minimally invasive, nonlethal technique to assess contaminant burden. We collected caudal scutes from 96 Morelet's Crocodiles in Belize over 2 yr to analyze scute tissues for OCs and to compare the observed OC concentrations among different scute tissue (fat, cartilage, and muscle) and among crocodiles of different age classes, sexes, and collection locations. Organochlorines of the DDT-type subclass were detected in 72 of 96 crocodiles, with methoxychlor detected in all 72 scutes containing OCs and p,p-DDE, p,p'-DDT, and p,p-DDD detected in 54, 47, and 20 scute samples, respectively. Organochlorines were more-frequently detected in scutes of adult crocodiles, but methoxychlor was occasionally observed in juveniles at concentrations two orders of magnitude higher than observed in adults, suggesting maternal offload of methoxychlor to offspring, greater exposure through juvenile habitat and diet, or both. Organochlorines were detected in crocodiles from all sampling locations with more frequent and higher concentrations observed in crocodiles from lagoon habitats than from river habitats. This study demonstrates that scutes can be used as a nonlethal indicator of OCs present in Morelet's Crocodiles, a finding which has applications for determining the trophic transfer of $\mathrm{OC}$ pesticides through tropical aquatic food webs and for estimating the continuing risk posed to crocodiles and other species by OC pesticides.
\end{abstract}

Many chemical compounds that have been banned for decades in most industrialized countries still are commonly used throughout Central America. These include OC pesticides such as DDT which, despite limited availability after production facilities in Mexico ceased operation in 1999, has seen continued use in Belize for vector control (Alegria et al., 2000; Grieco et al., 2000; Roberts et al., 2002) and likely also for agriculture (Rainwater, 2003). The wide use and environmental occurrence of the OCs in Belize is evident in the detection of these chemicals in the region's sediments (Wu et al., 2000; Somerville and Liebens, 2011) and ambient air (Alegria et al., 2000; Shen et al., 2005; Wong et al., 2009). This indicates a great potential for exposure of these chemicals to wildlife in the region (Rainwater et al., 2007). Organochlorines tend to bioaccumulate in the food chain because of their environmental persistence and their propensity for storage within fat tissues, causing high toxicological risk in top predators because of biomagnification $(\mathrm{Wu}$ et al., 2000; Ridal et al., 2001). General effects of OCs can include immunosuppressive effects, reproductive and developmental effects, cancer and tumor formation, and responses at elevated biochemical concentrations (Longnecker et al., 1997). Crocodilians may be at increased risk of adverse exposure and effects of OC pesticides because of their long life span, their trophic status as top predators, and because OC pesticides have been heavily used and persist in their tropical aquatic habitats.

Morelet's Crocodiles (Crocodylus moreletii) occupy freshwater habitat in the Atlantic and Caribbean lowlands of Mexico, Guatemala, and Belize. Morelet's Crocodiles were formerly listed as an endangered species but hunting restrictions put in place in the 1970s have resulted in populations recovering to the point where Morelet's Crocodiles are now classified as a species of least concern (Cedeño-Vázquez et al., 2012). Morelet's have

${ }^{2}$ Corresponding author. E-mail: brie.sherwin@ttu.edu

DOI: $10.1670 / 14-029$ potential for use as a sentinel species for environmental effects of OC pesticides because of their long life and ecological position. Several studies of OC burdens in crocodilians from Central America and the United States have utilized noninvasive techniques to collect data on residue concentrations including the use of nonviable eggs and chorioallantoic membranes (CAMs) from hatched eggs (Pepper et al., 2004; Rauschenberger et al., 2004). Such studies have established the presence of OCs in eggs of Morelet's Crocodile (Wu et al., 2000; Pepper et al., 2004) and generally in crocodilians from various locations across the globe (Phelps, 1996; Milnes et al., 2004; Wu et al., 2006; Rainwater et al., 2007, 2008). Although the quantification of OCs within nonviable eggs and CAMs of hatched eggs may be useful for assessing relative exposure to OC pesticides among crocodiles from different sites, egg-based OC concentration measures can be limited because concentrations do not necessarily equate with the total body burden of OC contaminants in adult crocodiles, cannot be easily linked to individual crocodiles, and provide no information about the presence/absence of OCs within adult male crocodiles (Pepper et al., 2004). Analyzing the OC concentrations within crocodile caudal (tail) scutes has been suggested as a minimally invasive technique for estimating crocodile OC body burdens at the individual scale (Rainwater et al., 2007); however, there has been no comprehensive study to date focused on testing OC residues found in crocodile scute tissues.

Crocodilian caudal scutes are triangular, vertically extended, laterally flattened dermal scales found on the dorsal surface of the tail (Richardson et al., 2002). Historically, dorsal caudal scutes of crocodiles have been used as a marking technique in wildlife management programs to obtain information on migration, behavior, age validation, mortality, and population size (Jennings et al., 1991). This minimally invasive, nonlethal technique has historically been used as an effective tool to qualitatively assess the mercury contaminant burden in 